\title{
Prognostic Role of Preoperative Circulatory Immune Cells and ratio of Neutrophil-to-Lymphocyte, Platelet-to-Lymphocyte and Lymphocyte-to- Monocyte in Women with Breast Cancer of Saurastra, Gujarat Region
}

\author{
Priya Mehta ${ }^{1}$, Girish K Goswami ${ }^{2}$, Nimesh Rupala ${ }^{3}$ \\ ${ }^{1,2}$ Dept. of Microbiology and Biotechnology, C U Shah Institute of Life Sciences, Wadhwan City, Surendranagar, India \\ ${ }^{3}$ Department of Biotechnology and Bioinformatics, La Trobe University, Melbourne, Australia \\ *Corresponding author : girishkgoswami@gmail.com, Mobile : +91 8320963005
}

Available online at: www.isroset.org

Accepted: 25/Aug/2018, Online: 30/Aug/ 2018

\begin{abstract}
Cancer is one of the frequently talked and most feared diseases that have evolved, rather rapidly in the past two decades. Breast cancer, a multifactorial disease is the second most common malignancy related mortality among women worldwide. Routine diagnosis for breast cancer is usually done by mammography and histopathology, but current research data suggest that other non-disease specific biomarkers such as protein profiling, tumor specific hormones, hematocrit, serum proteins etc are also hypothesized to be associated with overall health outcome of the patients and can serve as a supplementary parameter to predict overall prognosis of the patients. The present study concerns about relative analysis of Hematocrit and WBC parameters among patients at the time of diagnosis along with the examination of ratio of Neutrophil-to-Lymphocyte (NLR), Platelet-to-Lymphocyte (PLR) and Lymphocyte-to-Monocyte (LMR) by fully automated clinical biochemistry analyzer and its correlation with other socio-demographic factors prevalent among the patients. For this purpose, preoperative blood samples of breast cancer patients were collected from Rajkot Cancer Hospital and results were compared with age and sex matched healthy volunteers. The present study conclude that high NLR ratio in 27\%, high PLR ratio in $7.81 \%$ and low LMR ratio in $14.75 \%$ of patients was obtained among the studied population.
\end{abstract}

Keywords- Breast Cancer, Socio-demographic, WBC, NLR, PLR, LMR, prognosis

\section{INTRODUCTION}

As per the WHO (World Health Organization 2011) report, rather than coronary heart disease or stroke, cancer now causes more deaths worldwide and over 20 million new cancer cases is expected by 2025 [1]. In-spite of improvement in detection and treatment methods, breast cancer is the commonest malignancy diagnosed in women throughout the world and its incidence are increasing day by day $[2,3]$.

Cancer is a major public health challenge in India too and recent data from ICMR (Indian Council of Medical Research) estimated that there are nearly 1.5 lakh new breast cancer cases reported during 2016. The prognosis of patients depends on biological characteristics of the cancer and on the general conditions of patient together with the type of treatment being carried out. Established prognostic and predictive markers for breast cancer are age, tumour size, grade, nodal status, and endocrine/Her2neu receptor status [4].
Although TNM (Tumor, Nodes, and Metastasis) staging and lymph node status is considered the most important and widely used prognostic marker, the overall behavior of breast cancers is unpredictable with significantly different clinical outcomes of the treatments from patients with the same lymph node status and staging [5].

As hypothesized the overall behavior of cancer involves complex interplay between numerous molecular cascades and processes, it is recognized that inflammation too plays a major role in this cross talk as a hallmark of cancer biology. Chronic inflammation has been suggested to be a critical component of tumor progression, metastasis and relapse [6]. A multi-factorial network of chemical signals is initiated in response to injury which involves the activation and controlled migration of leucocytes (neutrophils, monocytes and eosinophils) from the circulatory system to the site of damage [7].

Neutrophil and platelet count plays an important role in the progression of several solid tumors. Research data also suggests that the assessment of hematologic parameters of the systemic inflammatory response may determine severity, 
mortality and treatment follow-up in case of breast cancer patients [8, 9].

Apart from the traditional diagnosis, current need is to have a non invasive and rapid method of detection and early evaluation of the overall prognosis of the breast cancer patients. Considering the probable role of the hematological parameters in progression of breast cancer and their usability as a prognostic marker for early metastasis detection, this study was designed with an principal objective to determine the relationship between serum levels of neutrophil, lymphocyte, monocyte and platelets and their ratios at the time of initial diagnosis together with the staging and grading in breast cancer patients.

\section{MATERIALS AND METHODS}

In the present study we retrospectively identified newly diagnosed and histologically confirmed primary Breast Cancer patients admitted at Rajkot Cancer Society Hospital for breast cancer surgery and treatment from December 2016 to May 2017. Preoperative blood samples were collected from the newly diagnosed breast cancer patients and complete blood profiling was performed with an aim to evaluate the relative percentage and ratio of Neutrophil, Monocytes, Lymphocyte and Platelets and the obtained results were compared with other clinic-pathological and socio-demographic data of the to rule out overall health status of the breast cancer patients.

Total 71 patients meet the inclusion criteria of having a complete blood count with leukocyte differential count performed before initiating any treatment. Data for most of the patients $(95 \%)$ were collected prior to any surgical intervention (either mastectomy or conservative breast surgery). Exclusion criteria for the study include clinical evidence of active infection, presence of hematological disorders, acute as well as chronic inflammatory or autoimmune diseases, or prior steroid therapy. Patients who had received any treatment before surgery or neoadjuvant chemotherapy or who had recurrence or metastatic disease were not included in this study.

A detailed clinical history for each of the patient was recorded on a standardized questionnaire designed and approved for the study. History of menstruation period, pregnancy, previous inflammation or infection, usage of oral contraceptives, hormonal medications, prior surgery and use of other traditional medicine along with family history of cancer, obesity, hypertension or disease such as diabetes, was recorded. Addiction to smoking, drinking alcohol and chewing tobacco and their area of belonging region was also recorded.

The whole blood samples of all the included breast cancer patients were collected in vaccunated EDTA tube with $20 \%$
EDTA as anti coagulant, under sterilized condition from patients prior to initiation of any treatment. They were also informed that their samples will only be used for research purpose and their confidentiality will be maintained.

Further complete blood count analysis together with differential count was carried out on the same day of the blood collection using automated hematological analyzer from Meril Diagnostics. The neutrophil, lymphocyte, monocyte and platelet counts were recorded, and the NLR, the PLR and the LMR were calculated. This study was approved by the Ethics Committee and the Institutional Review Board of the Hospital and the college and all the Breast Cancer patients were from the Saurastra region of Gujarat.

Statistical Analysis. Statistical analysis and correlation study between different parameters was carried out using JAVA STAT 2 software, Microsoft Office Excel 2007 and Graph Pad Prism 5 software.

\section{RESULTS}

Total 100 newly diagnosed and histologically confirmed Breast Cancer patients were undergone primary preoperative screening, out of which the 71 patients were included in this study and remaining 29 were excluded based on exclusion criterion. The median age of the patients is $50.13 \pm 11.59$ years (range : $30-80$ years). The mean values of white blood cells, Platelets, Neutrophils, Lymphocytes and Monocytes were $7.77 \times 10^{9}$ cells/L (range, 2.34-15.76 $\times 10^{9}$ cells/L), 328.61 cells $/ \mathrm{L}$ (range, 50-528 cells/L), 5.1 x $10^{9}$ cells/L (range, $0.93-12.11 \times 10^{9}$ cells $/ \mathrm{L}$ ), $2.13 * 10^{9}$ cells/L (range, 1 $3 \times 10^{9}$ cells $/ \mathrm{L}$ ) and $0.34 \times 10^{9}$ cells $/ \mathrm{L}$ (range, $0.01-2.39 \times 10^{9}$ cells/L). The threshold values of NLR, PLR and LMR were used directly from the standard quoted references, which was been determined by ROC (Receiver Operating Characteristic) Curve analysis. The baseline characteristics of the Breast Cancer Patients are summarized in Table 1.

Table 1 : Clinico-Pathological Data of Breast Cancer Patients.

\begin{tabular}{|lll|}
$\begin{array}{l}\text { Baseline Characteristics of } \\
\text { the Breast Cancer Patients }\end{array}$ & $\begin{array}{l}\text { No. of } \\
\text { Patients }\end{array}$ & $\begin{array}{l}\text { Percentage } \\
\%\end{array}$ \\
\hline Total Patients & 71 & 100 \\
\hline Age Groups & 12 & \begin{tabular}{l} 
Mean +- SD \\
\hline$<40$ Years
\end{tabular} \\
\hline$>40$ Years & 59 & $53.34 \pm 2.27$ \\
\hline Menopausal Status & & \\
\hline Pre Menopausal & 29 & $40.85 \%$ \\
\hline Post Menopausal & 40 & $56.34 \%$ \\
\hline Amenorrhea & 1 & $1.41 \%$ \\
\hline Male Patient & 1 & $1.41 \%$ \\
\hline
\end{tabular}




\begin{tabular}{|c|c|c|}
\hline \multicolumn{3}{|l|}{ Background of Patients } \\
\hline Urban Region & 31 & $43.66 \%$ \\
\hline Rural Region & 40 & $56.34 \%$ \\
\hline \multicolumn{3}{|l|}{ Type of Breast Cancer } \\
\hline Ductal Carcinoma In-Situ & 17 & $28.81 \%$ \\
\hline $\begin{array}{l}\text { Invasive/Infiltrating Ductal } \\
\text { Carcinoma }\end{array}$ & 40 & $67.80 \%$ \\
\hline Phyllodes Tumor & 1 & $1.69 \%$ \\
\hline Papillary Carcinoma & 1 & $1.69 \%$ \\
\hline $\begin{array}{l}\text { Type of Treatment } \\
\text { undertaken by Confirmed } \\
\text { Breast Cancer Patients }\end{array}$ & & \\
\hline Surgery & 44 & $80 \%$ \\
\hline Chemotherapy & 1 & $1.82 \%$ \\
\hline Surgery + Chemotherapy & 9 & $16.36 \%$ \\
\hline Traditional medicine & 1 & $1.82 \%$ \\
\hline $\begin{array}{l}\text { Prior Clinical Familial } \\
\text { History in Breast Cancer } \\
\text { Patients }\end{array}$ & 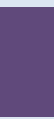 & \\
\hline Surgery & 48 & $67.61 \%$ \\
\hline Inflammation & 22 & $30.99 \%$ \\
\hline Cancer & 13 & $18.31 \%$ \\
\hline $\mathrm{BP}$ & 14 & $19.72 \%$ \\
\hline TB & 5 & $7.04 \%$ \\
\hline Diabetes & 12 & $16.90 \%$ \\
\hline Obesity & 8 & $11.27 \%$ \\
\hline Addiction & 15 & $21.13 \%$ \\
\hline
\end{tabular}

Histologically confirmed 71 breast cancer patients were included in this study, out of which 31 patients were from urban region and 40 patients were from rural region. The obtained results indicates that there is high prevalence of breast cancer among rural background region due to lack of awareness and their presentation to the hospital is also in advance stage of the disease due to illiteracy and lack of resources.(graph not shown).

The 71 patients included in this study were divided into different age groups ranging from 30 to 80 years of age. The mean age of the patients at the time of study was $50.13 \pm 11.59$ years. Maximum numbers of patients are observed among the age group of $40-49$ years of age (Graph 1).

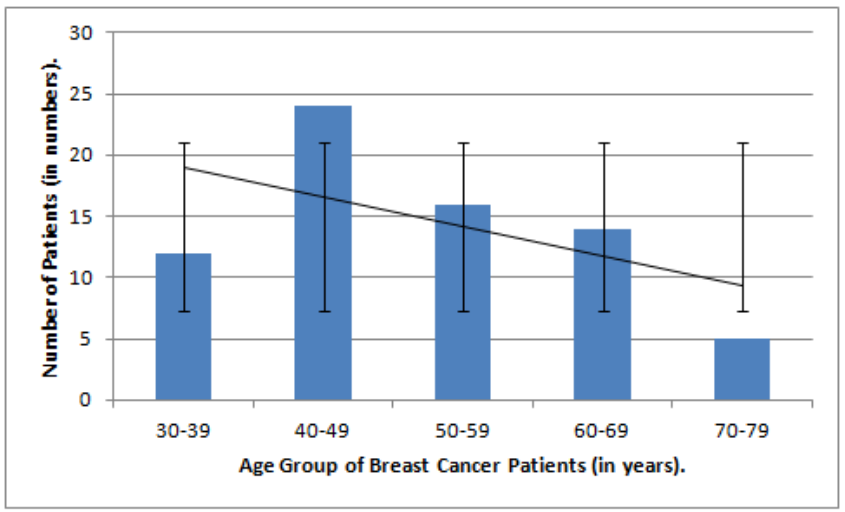

Graph 1: Age Wise Distribution Pattern of Breast Cancer Patients.

As per reports of several studies the risk of incidence of breast cancer increases with increase in age of female. The highest incident rate of breast cancer was observed among 40-49 years of age group followed by 60-69 years of age group. Out of total patients under this study majority cases were belonging to post menopausal status, few were in pre menopausal status and 1 patient had amenorrhea. These results indicate that the post menopausal status is associated with increased risk of breast cancer development as it leads to hormonal imbalance and altered metabolism in female body after stoppage of menstrual cycle. (graph not shown).

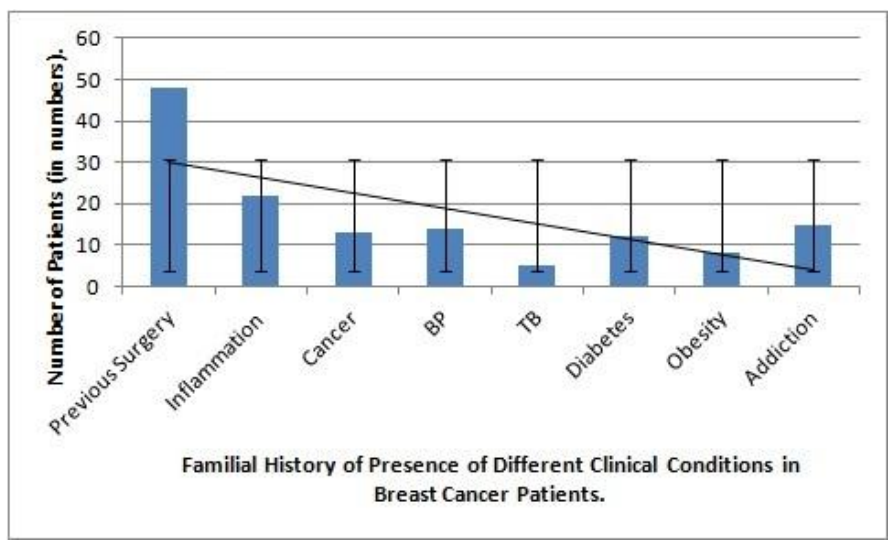

Graph 2: History of Presence of Several Familial Conditions in Breast Cancer Patients.

Cancer is a multifactorial disease and doesn't possess single risk factor; in this study it has been also found that many patients possess the history of different risk factors. There were 48 patients, who had undergone different types of surgery previously. Inflammation condition was found out among 22 patients. There were 13 patients out of total who were having family history of different types of cancer running from generations. Obesity was observed among 8 
breast cancer patients. 14 patients had a family history of blood pressure problem and proven mild dysfunction of heart. There were 5 cases among the patients, who had history of tuberculosis. 12 patients had history of diabetes mellitus. 15 patients were having different kinds of addictions like pan, betel nut, tobacco, snuff, smoking etc since years (Graph 2).

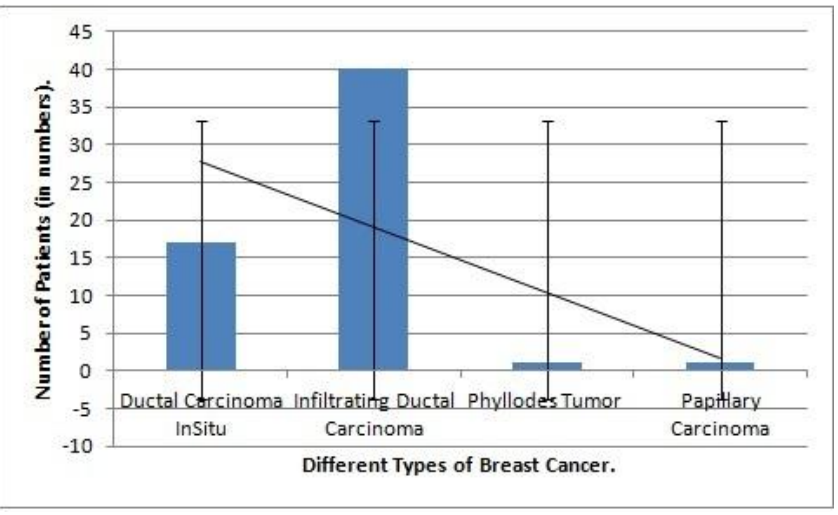

Graph 3: Prevalence Data of Different Types of Breast Cancer among Patients.

The patients enrolled in this study were suffering from different types of breast cancers with different staging and grading. There were 17 patients with Ductal carcinoma in situ and 40 patients with Invasive/Infiltrating ductal carcinoma. There was 1 case of Phyllode tumor and 1 case of papillary carcinoma. The patients included in the study were undergoing different types of treatments. There were 44 patients who had undergone only surgery, 1 patient who had received only chemotherapy and 9 patients who had received both surgery and chemotherapy. One patient was taking traditional medicine along with the routine treatment. There were missing data of 12 patients regarding the type of breast cancer and 16 patients regarding the type of treatment they received (Graph 3).

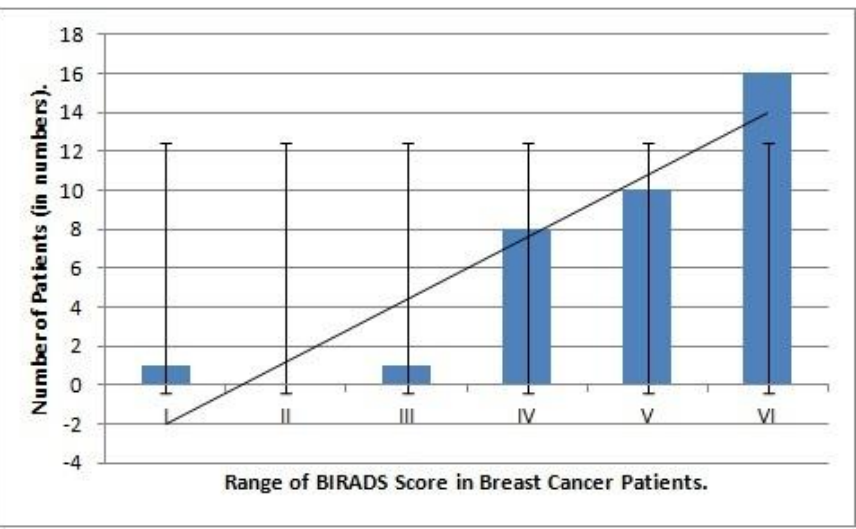

Graph 4: Range of BIRADS Score observed among Breast Cancer Patients.
The patients participated in this study were categorized according to their Birads (Breast Imaging-Reporting and Data System ) score in Sonomammography test which was done at the time of diagnosis. There was 1 patients with Birads score I which indicates negative findings. There was 1 patients with Birads III score which states that there is probability of benign with $<2 \%$ chances of malignancy. There were 8 patients with Birads score IV which suggests that there may be abnormality with $2-95 \%$ chances of malignancy. There were 10 patients with Birads score $\mathrm{V}$ which indicates that tumor is highly malignant with $>95 \%$ chances of malignancy and there were 16 patients with Birads score VI which suggests that there is malignancy proven by biopsy. Birads score was not available for 35 patients (Graph 4).

Out of total 71 subjects, during this study platelet count of 70 patients, neutrophil count for 63 patients, lymphocyte count for 64 patients and monocyte count for 61 patients were studied. The mean value of platelet count was found $328.61 \pm 90.14$, mean value of neutrophil count was found $5.09 \pm 1.63$, the mean value of lymphocyte count was found $2.13 \pm 0.84$ and the mean value of monocyte count was found $0.34 \pm 0.15$ respectively.

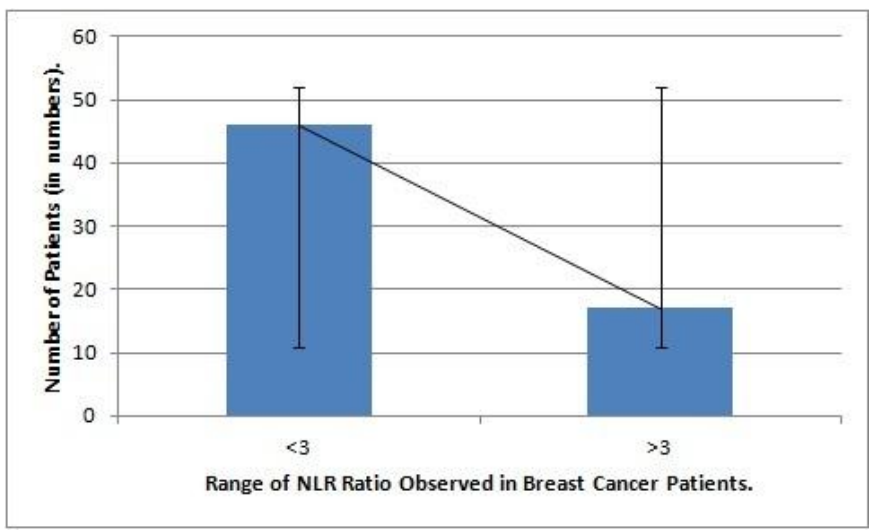

Graph 5: Range of NLR Ratio among Breast Cancer Patients.

Mean value of NLR of 63 patients was $2.92 \pm 2.69$ and as per the previous standard reference the NLR value $<3$ indicates good prognosis of disease where as NLR value $>3$ indicates poor DFS (Disease Free Survival) and OS (Overall Survival) [12]. There were 46 patients that had NLR value $<3$ and 17 patients had NLR value $>3$ which indicates that around $27 \%$ patients had higher value of NLR indicating poor DFS and OS of the breast cancer patients (Graph 5). 


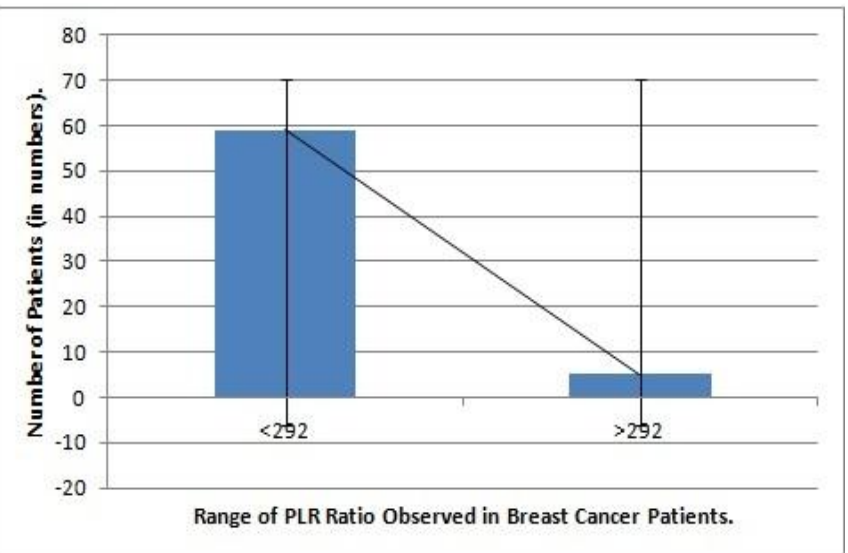

Graph 6: Range of PLR Ratio among Breast Cancer Patients.

Mean value of PLR of 64 patients was $184.81 \pm 147.07$ and as per the standard reference it was previously found out that PLR value <292 indicates good prognosis of the disease where as PLR value $>292$ indicates poor DFS and OS. There were 59 patients that had PLR value $<292$ and 5 patients had PLR value $>292$ which shows that $7.81 \%$ patients had higher value of PLR indicating poor DFS and OS of the breast cancer patients (Graph 6).

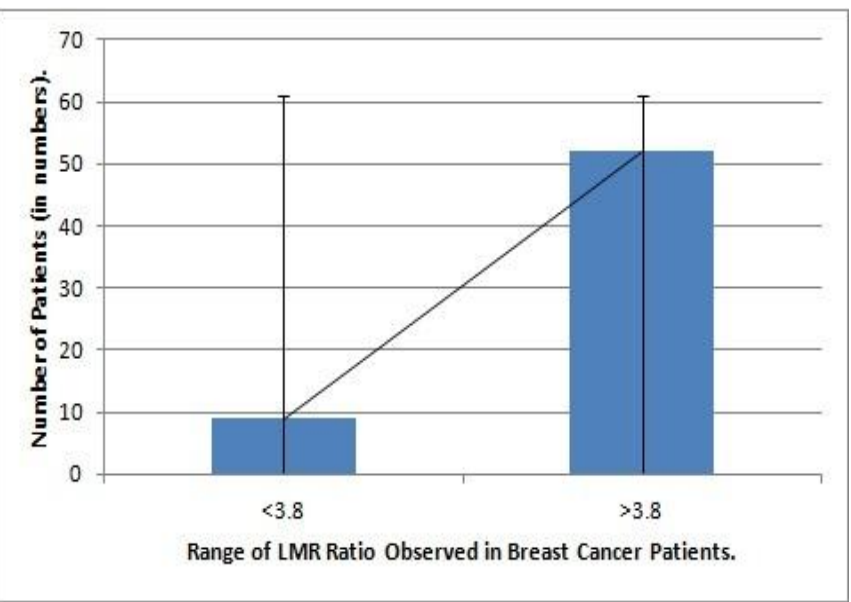

Graph 7: Range of LMR Ratio among Breast Cancer Patients.

Mean value of LMR of 61 patients was found out to be $7.88 \pm 6.05$ and as per the standard reference it had been found out that LMR value $<3.8$ indicates poor DFS and OS where as LMR value $>3.8$ indicates good prognosis of disease [13]. There were 9 patients that had LMR value $<3.8$ and 52 patients had LMR value $>3.8$ which indicates that $14.75 \%$ patients had lower value of PLR indicating poor DFS and OS of the breast cancer patients (Graph 7).

\section{DISCUSSION}

As per reports of several studies the risk of incidence of breast cancer increases with increase in age of female. The highest incident rate of breast cancer was observed among 40-49 years of age group followed by 60-69 years of age group. These results raise an important concern about change pattern of breast cancer incidence and increased number of patients at younger age with an aggressive phenotype of breast cancer. Similar findings were also reported with maximum numbers of breast cancer cases in the age group of 35 to 44 years of age and they also observed that the average age of cases of breast cancer was among mean age of 45.64 years $[10,11]$.

Breast cancer occurs due to malignancy in breast cells. The malignant cells arise within the lining of the milk glands or ducts of the breast and develop breast carcinoma [14]. Apart from all the subtypes, the burden of metastatic breast cancer is devastating and it is the leading cause of over half a million deaths of women worldwide, out of 1.3 million women who will be diagnosed each year [15]. This study was initiated with a hypothesis to rule out probable use of NLR, PLR and LMR ratios to predict chances of metastasis in the breast cancer patients based on their preoperative levels. Among the studied patients, high NLR in 27\%, and high PLR ratio in $7.81 \%$ was obtained, which indicates of poor prognosis whereas low LMR ratio in $14.75 \%$ of patients which is indicative of poor prognosis in the patients respectively.

In this study it was also found out that there is progressive increase in incidence of breast cancer among women at younger age. The result also indicates that rural regions have high incidence rate as compared to urban regions were healthcare facilities are easily available. It was also observed that early menstruation, late menopause, early age of marriage and child birth at younger age, infectious conditions such as $\mathrm{TB}$, familial history of cancer, previous surgery, hypertension and previous inflammation or infection are proving to be major risk factors for developing breast cancer in later age among the females.

Our study also suggests that NLR, PLR and LMR may be associated with DFS and OS of patients with breast cancer. These results suggest that cancer-associated inflammation might play a greater role in promoting breast cancer progression and can be easily introduced in clinical practice 
in order to identify breast cancer patients with poor prognosis.

\section{CONCLUSION AND FUTURE SCOPE}

The advantages of the analysis of these parameters are that they are simple, quick, and relatively cheaper and can be easily carried out in even smaller laboratories without facility of any sophisticated instruments. If fruitful results are obtained in larger cohort study, with a brief clinical history and detailed follow up of the patients, then it may offer a new and inexpensive opportunity to delineate women who are at high risk of metastatic breast cancer and provides an hope of targeting surveillance, allowing early detection of progression of disease and thereby reducing mortality occurring from this disease.

The major limitation of the study is that it is observational, single region specific and the sample size was relatively small to justify the effect of multiple clinical features on survival. So the obtained results could not be generalized to the other population. The prognostic value of these biomarkers' levels should be evaluated in a larger, multicenter design for verification and to understand the mechanism of systemic inflammation and its role in altering tumor microenvironment.

\section{ACKNOWLEDGMENT}

Deep Gratitude towards Management of Shree $M$ \& $N$ Virani Science College, Rajkot for necessary Approval and technical support for undertaking the experimental work with support of students Ms. Shivani jani, Ms. Deevya Chetnani, Ms. Devanshi Adhia, Ms. Shraddha Vala and special thanks to Dr. Neepa Pandhi and Dr. Minaxi Parmar for their guidance and support.

Necessary Samples and Brief Patient History was obtained from Rajkot Cancer Hospital through prior approval of ethical committee and special thanks to Dr. Gupta.

Conflict of Interest: The authors state no conflict of interest and have received no payment in preparation of this manuscript.

\section{REFERENCES}

[1]. Jacques Ferlay, Isabelle Soerjomataram, Rajesh Dikshit, Sultan Eser, Colin Mathers, Donald Maxwell Parkin, David Forman and
Freddie Bray. " Cancer incidence and mortality worldwide: Sources, methods and major patterns in GLOBOCAN 2012 ". International Journal of Cancer, Volume 136 Issue 5 : E359-86, March 2015.

[2]. Smita Asthana, Sonia Chauhan, Satyanarayana Labani.. “ Breast and cervical cancer risk in India: An update ". Indian Journal of Public Health, Volume 58, Issue 1 : 5-1, 2014.

[3]. S Krenn-Pilko, U Langsenlehner, E-M Thurner, T Stojakovic, M Pichler, A Gerger, K S Kapp and T Langsenlehner. "The elevated preoperative platelet-tolymphocyte ratio predicts poor prognosis in breast cancer patients ". British Journal of Cancer, Issue 110, pp. 2524-2530, 2014.

[4]. J Kraeima, S Siesling, IM Vliegen, JM Klaase, MJ Ijzerman. “ Individual risk profiling for breast cancer recurrence: towards tailored follow-up schemes ". British Journal of Cancer, Issue 109, pp. 866-871, 2013.

[5]. S. Eva Singletary, James L. Connolly. " Breast Cancer Staging: Working With the Sixth Edition of the AJCC Cancer Staging Manual ". CA Cancer Journal of Clinician, Volume 56, Issue 1, pp. 37-47, 2006.

[6]. FR Balkwill, A Mantovani. " Cancer-related inflammation: common themes and therapeutic opportunities ". Seminars in Cancer Biology, Volume 22, Issue 1, pp. 33-40, 2012.

[7]. Yoo Young Lee, Chel Hun Choi, Ha Jeong Kim, Tae Joong Kim, Jeong Won Lee, Je Holee, Duk Soo Bae and Byoung Gie Kim. “ Pretreatment neutrophil: lymphocyte ratio as a prognostic factor in cervical carcinoma ". AntiCancer Research, Volume 32, Number 4, 2012.

[8]. S Kilin calp, S Coban, H Akinci, M Hamamc, F Karaahmet, Y Coskun . " Neutrophil/lymphocyte ratio, platelet/lymphocyte ratio, and mean platelet volume as potential biomarkers for early detection and monitoring of colorectal adenocarcinoma ". European Journal of Cancer Prevention, Volume 2, Number 4 : 328-33, July 2014.

[9]. J Szkandera, A Gerger, B Liegl-Atzwanger, G Absenger, M Stotz, H Samonigg, W Maurer-Ertl, T Stojakovic, F Ploner, A Leithner, M Pichler. "Validation of the prognostic relevance of plasma Creactive protein levels in soft-tissue sarcoma patients ". British Journal of Cancer., Volume 109 : pp. 2316-2322, 2013.

[10]. Navneet Kaur, Amit Attam, Sudipta Saha, S. K. Bhargava. “ Breast Cancer Risk Factor Profile in Indian Women ". JIMSA., Volume 24, Number 4, 2011.

[11]. Ramchandra Kamath, Kamaleshwar S Mahajan, Lena Ashok, and T S Sanal. " A Study on Risk Factors of Breast Cancer Among Patients Attending the Tertiary Care Hospital, in Udupi District". Indian Journal of Community Medicine, Volume 38, Issue 2, pp. 95-99, 2013.

[12]. Mirco Pistelli, Mariagrazia De Lisa, Zelmira Ballatore, Miriam Caramanti, Alessandra Pagliacci, Nicola Battelli, Francesca Ridolfi, Matteo Santoni, Elena Maccaroni, Raffaella Bracci, Alfredo Santinelli, Tommasina Biscotti, Rossana Berardi and Stefano Cascinu. "Pre treatment neutrophil to lymphocyte ratio may be a useful tool in predicting survival in early triple negative breast cancer patients ". BMC Cancer, Volume 15, Issue 195, pp. $1-9,2015$

[13]. Jiahuai Wen, Feng Ye, Xiaojia Huang, Shuaijie Li, Lu Yang, Xiangsheng Xiao, and Xiaoming Xie. "Prognostic Significance of Preoperative Circulating Monocyte Count in Patients With Breast Cancer Based on a Large Cohort Study". Medicine., Volume 94, Number 49, pp. 1-7, December 2015.

[14]. Christian Nordqvist. " Breast Cancer: Causes, Symptoms and Treatments". Medical News Today, 2017.

[15]. Musa Mayer. "Lessons Learned From The Metastatic Breast Cancer Community ". Seminars in Oncology Nursing, Volume 26, Issue 3, pp. 195-202, 2010.

[16]. Centers for Disease Control and Prevention (CDC) data. 
[17]. Indian Council of Medical Research (ICMR) 2016 report.

[18]. National Institute of Cancer Prevention and Research (NICPR) epidemiology data.

\section{AUTHORS PROFILE}

PRIYA MEHTA had completed M.Sc (Integrated) Medical Biotechnology from ARIBAS, New VV Nagar, Anand, Gujarat, India (2013). She has qualified GATE Biotechnology Examination during the year 2014 conducted by IIT and also qualified BAT examination with AIR $8^{\text {th }}$

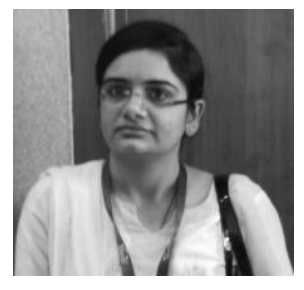
Rank and BULATS Examination conducted by SCOPE and Cambridge University. Posses research Experience from GCRI Ahmedabad, M S University - Baroda and ICIRC - Ahmedabad. Possess Academic Experience from Shree M \& N Virani Science College, Rajkot and C U Shah Institute of Life Sciences, Surendranagar. She is now doing Ph.D research in the area of Cancer Biology from C U Shah University, Surendranagar.

Girish K Goswami Girish K. Goswami, Dean Faculty of Science, C U Shah University, Wadhwancity is an academician and researcher with more than sixteen years of work experience in the field of molecular biology/biotechnology. After completing

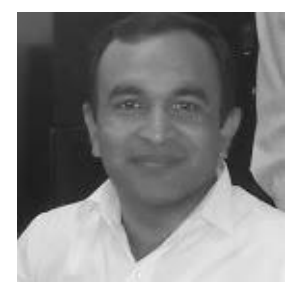
his master in Biotechnology form IIT Roorkee, India, he worked at NIMS Medical College, Jaipur followed by JECRC and Amity University Rajasthan, Jaipur respectively. He has published more that 17 international research articles and filled 3 patents to Indian Patent Office, New Delhi. He has developed programs in Microbiology and Biotechnology at B. Tech. (Biotech), B. Sc., M. Sc., M. Phil. and Ph. D. levels.

Nimesh Rupala had completed three years graduation in B.Voc MLMDT from Shree M \& N Virani Science College, Rajkot, Gujarat, India. He is currently pursuing an Master degree in Biotechnology and Bioinformatics from College of Science, Health and Engineering from La Trobe University,

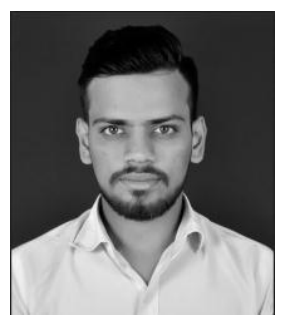
Australia. 